\title{
Fate of Forgotten DJ Stent
}

\section{Bikash Bikram Thapa, Narayan Thapa and Bharat Bahadur Bhandari}

Department of Surgery, Nepalese Army Institute of Health Sciences, Shree Birendra Hospital, Chhauni, Kathmandu, Nepal

\section{ABSTRACT}

Introduction: Double J stent is one common armamentarium used in urological procedure. It can serve both therapeutic and prophylactic function. However the use of double $\mathrm{J}$ stent is invariably associated with minor to some of major complications. Forgotten DJ stent is one untoward issue of stenting patient that is largely preventable and entails complex urological procedure to remove it.

Methods: We performed descriptive study where data were collected prospectively from patients who have double J ureteral stent in situ after urological procedure. Stent left more than three months was defined as forgotten DJ stent. Demographic characteristics, clinic-radiological data and details of management were noted to evaluate the mode of presentation, associated complications and mode of treatment. The reason behind forgetting those stent was asked with study population and presented.

Results: The total of 27 cases of forgotten DJ stent cases were recorded during period of December 2013 to January 2018. Mean age of patient was $46.6 \pm 12.25$ years. The longest indwelling time was 10 years. Stent syndrome was common mode of presentation followed by encrustation. Majority $(92.5 \%)$ of the patients were managed with endourological approach and $26 \%$ (7) of cases required more than one modalities of treatment.

Conclusions: The forgotten stent is an avoidable condition through proper patient counselling. When required the management necessitates simple cystoscopic to complex endourolgical intervention.

Key words: double J stent; endourological approach; stent syndrome

Correspondence: Bikash Bikram Thapa, Department of Surgery, Nepalese Army Institute of Health Sciences, Shree Birendra Hospital, Chhauni, Kathmandu, Nepal. Email: bbthapa@gmail.com

DOI: $\underline{\text { http://dx.doi.org/10.3126/mjsbh.v17i2.19901 }}$

Submitted on: 2018-05-13

Accepted on: 2018-06-29 


\section{INTRODUCTION}

The use of indwelling stent is a routine practice in upper urinary tract surgery. First clinical application of ureteral stent was reported in 1967 by Zmskind and later in 1970.1,2 In 1972 Goodwin defined stent as mould for internal fixation which provides effective uninterrupted internal urinary drainage from kidney to bladder, reduce or eliminate urinary leakage and provide ureteral stenting. 3,4

JJ stents are usually made from silicon or polyurethane. Studies demonstrated two to four weeks as standard indwelling time for such ureteral stent. ${ }^{5}$ The characteristics of the ideal ureteric stent was elaborated by Denstedt et al. in $1943 .{ }^{6}$ Different complications can occur with the short or long term use of indwelling stents. ${ }^{7-9}$ A forgotten DJ ureteric stent can cause a spectrum of complications ranging from hematuria, stent occlusion, migration, fragmentation, encrustation, and stone formation. Serious complications like urosepsis, renal failure, fistula formation with iliac arteries and even morality have been reported due to forgotten DJ stent. Studies consider variable period of more than three to six months to call it as forgotten stent. 10 We discussed here management of 27 cases of forgotten DJ stent which otherwise get neglected most of the time.

\section{METHODS}

This is a retrospective study where data were collected prospectively involving the patients who underwent double $\mathrm{J}$ ureteral stenting for various urological indications. Patients were enrolled in the study at the time of stent removal appointment after written consent. The duration of stent placement was noted. Stent dwelling time more than three months were considered as forgotten DJ stent. Patient's awareness regarding the indwelling DJ stent was noted. Complete blood count, renal function test, urine routine and microscopic examination, urine culture sensitivity was done in all cases before removal of DJ stent. All patients were evaluated for stent encrustation and associated stone burden with KUB and non-contrast enhanced stone protocol CT and/or IVP. Diethylenetriamine pentaacetic acid (DTPA) renography was performed to estimate renal function in selected case. Prophylactic antibiotic (third generation cephalosporin) was given. The management is planned depending upon the details of encrustration and the stone burden.

For stents with no encrustation gentle nontraumatic retrieval was attempted through 21 French rigid cystoscope under local anaesthesia. Ureterorenoscopy (URS) was performed using a $6.5 \mathrm{Fr} / 45 \mathrm{~cm}$ semirigid ureteroscope under fluoroscopic guidance. Percutaneous nephrolithotomy (PCNL) was carried out using an 9/11.5 Fr , $25 \mathrm{~cm}$ semirigid urteroscope or $24 \mathrm{Fr}$ rigid nephroscope. Data were analysed with Microsoft excel 2007 and presented.

\section{RESULTS}

We encountered 27 cases of forgotten DJ stent cases during period of December 2013 to January 2018. Mean age of patient was $46.6 \pm 12.25$ years out of which 16 were male and 11 were female. The indwelling time was four months to 10 years. 22

Table 1. Management details of forgotten DJ

\begin{tabular}{|r|l|r|}
\hline SN & Operative procedure & Number \\
\hline 1 & Cystoscopic removal & 15 \\
\hline 2 & Cystolithotripsy + PCNL & 3 \\
\hline 3 & URSL +PCNL & 3 \\
\hline 4 & URSL & 2 \\
\hline 5 & Cystolitholapaxy & 2 \\
\hline 6 & Cystolithotomy + & 1 \\
\hline & Pylelolthotomy & Simple Nephrectomy \\
\hline
\end{tabular}




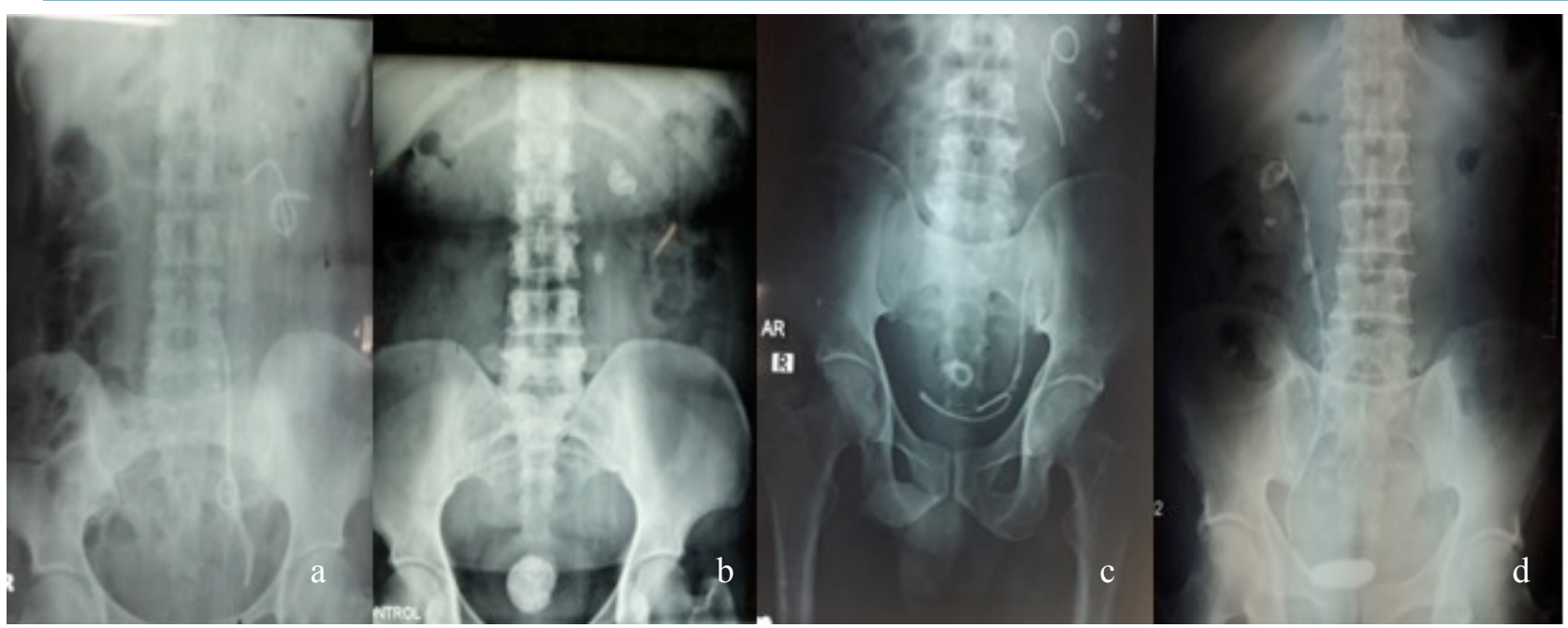

Fig 1. KUB images of forgotten stents (a) One year (b) Three years (c) Seven years (d) 10 years

patients presented to us within one year and five patients reported after one year. Stent syndrome was mode of presentation in $74 \%$ followed by encrustation or stone formation in $29.5 \%$ and urinary tract infection in $15 \%$. Urinary bladder was the common site of encrustation (59.2\%). We used multimodality approach for management of the forgotten stents (Table 1). Majority (92.5\%) of the patients were managed with endourological approach and $26 \%$ (seven) of cases required more than one modalities of treatment.

\section{DISCUSSION}

The double $\mathrm{J}$ ureteral stent allows direct internal drainage of upper urinary tract to the urinary bladder. The common indications are prophylactic stenting and relief of obstructive uropathy. ${ }^{4,12}$ The overall compilations rate ranges from $70 \%$ to $79.2 \% .{ }^{13}$ A forgotten DJ stent can cause spectrum of complications due to increasing encrustation and stone burden that happens mostly due to poor

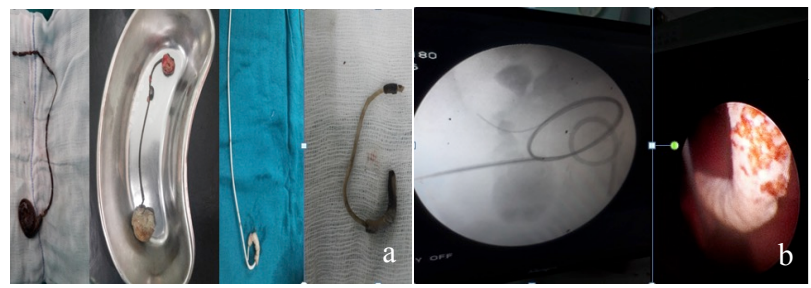

Fig 2. (a) Retrieved forgotten DJ stent with encrustrations (b) PCNL patient compliance and or inadequate counselling by the physicians. Urinary tract infection, indwelling time, bio-incompatibility, malignancy and metabolic abnormalities has positive correlation with stent encrustation. ${ }^{11,14}$ A study by el-Faqih et al. states "the stent encrustation rate increases from $9.2 \%$ for an indwelling time of less than six weeks to $47.5 \%$ for six to 12 weeks and $76.3 \%$ for more than 12 weeks." 15

The presentation of forgotten stent varies. Damiano et al. observed flank pain in $25.3 \%$, encrustations in $21.6 \%$, irritative bladder symptoms in $18.8 \%$, hematuria in $18.1 \%$, UTI in $12.3 \%$, and stent

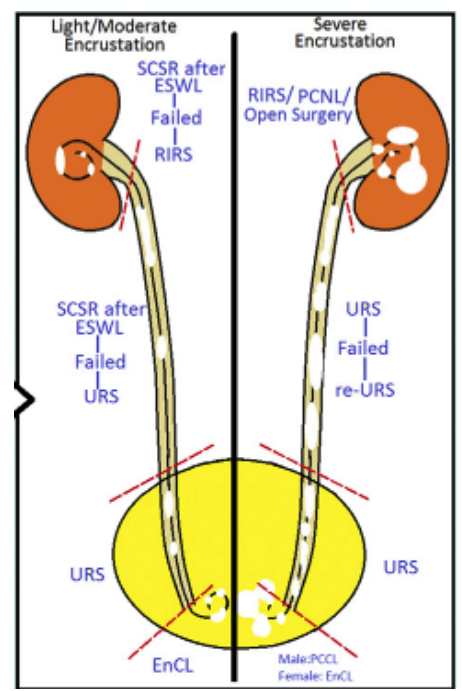

Fig 3. Management algorithm of encrusted DJ stent. 11 
migration in $9.5 \%$ of the patients. ${ }^{13}$ Stent syndrome that comprises flank pain, suprapubic discomfort, irritative voiding symptoms and occasional hematuria is common among our study populations. Sohreb et al. reported forgotten DJ stent as old as 23 years with $14.2 \%$ mortality among the 28 study subjects in 13 years. ${ }^{10}$ The management of forgotten DJ stent is challenging and mandates complex endourological intervention or open surgery ${ }^{16,17}$ Sancaktutar et al. has illustrated the management algorithm of encrusted DJ stent with multimodality approach depending upon the site and severity of encrustation and available resources (Fig. 3). ${ }^{11}$ Among our study populations $37 \%$ of the patients were unaware of the stent inside their body, $33 \%$ confused drain tube with DJ stent and 30\% patient was noncompliant with the follow up. Primary prevention can be done with judicious use of DJ ureteral stent. El Harrech et al. in his randomised controlled trial concluded that "Uncomplicated ureteroscopy for distal ureteral calculi without intraoperative ureteral dilation can safely be performed without placement of a ureteral stent."18 A systematic review and meta analysis published in Journal of Endourology in 2018 states that "Compared with double $\mathrm{J}$ stent, externalised ureteral catheter is also an effective alternative for patients with upper urinary stones in tubeless PCNL and could help patients reduced stent-related discomfort and avoided cystoscopy for DJS removal." 19 Prevention is the best management that can be attained with patient counselling, data recording and tracking. Study has reported the decline of incidence of forgotten stent from $12.5 \%$ to $1.2 \%$ with the use of computer based tracking software. ${ }^{3}$

\section{CONCLUSIONS}

A forgotten DJ stent invariably results in some type of complications that requires complex and multimodality approach for management. The judicious use, patient counselling and data recording can help to prevent the untoward effects of forgotten DJ stent.

To cite this article: Thapa BB, Thapa N, Bhandari BB. Fate of forgotten DJ stent. MJSBH. 2018;17(2): $38-43$.

\section{Conflict of Interest: None declared}

\section{REFERENCES}

1. Zimskind PD, Fetter TR, Wilkerson JL. Clinical use of long-term indwelling silicone rubber ureteral splints inserted cystoscopically. The Journal of urology. 1967 May;97(5):840-4.

PMID: 6025928

2. Marmar JL. The management of ureteral obstruction with silicone rubber splint catheters. J Urol. 1970 Sep;104(3):386-9.

PMID: 5459972

3. Lawrentschuk N, Russell JM. Ureteric stenting 25 years on: routine or risky? ANZ J Surg. 2004 Apr; 74(4):243-7.

DOI: http://10.1111/j.1445-2197.2004.02947. 
4. Nawaz H, Hussain M, Hashmi A, Hussain Z, Zafar N, Naqvi A, et al. Experience with indwelling "J.J ureteral stents. J Pak Med Assoc. 1993 Aug;43(8):147-9.

PMID: 8264090

5. Kumar R, Mandhani A, Kumar A, Ahlawat R. Optimum duration of splinting after endopyelotomy. J Endourol. 1999;13(2):89-92.

DOI: https://doi.org/10.1089/end.1999.13.89

6. Denstedt JD, Reid G, Sofer M. Advances in ureteral stent technology. World J Urol. 2000 Sep;18(4): $237-42$.

DOI: http:// 10.1007/s003450000180237.345

7. El Khader K. Complications of double J ureteral stents. Journal d'urologie. 1996;102(4):173. PMID: 9091568

8. Ringel A, Richter S, Shalev M, Nissenkorn I. Late complications of ureteral stents. Eur Urol. 2000 Jul; 38(1):41-4.

PMID: 10859440

9. Vallejo JH, Burgos FR, Alvarez JA, Sáez JG, Téllez MM, de la Muela Sánchez P. Double J ureteral catheter. Clinical complications. Archivos espanoles de urologia. 1998 May;51(4):361-73.

PMID: 9656558

10. Sohrab A, Aneesh S, Sureka SK, Varun M, Nitesh P, Manoj K, et al. Forgotten Reminders: an Experience with Managing 28 Forgotten Double-J Stents and Management of Related Complications. Indian J Surg. 2015 Dec;77(3):1165-71.

DOI: http://10.1007/s12262-015-1229-4

11. Sancaktutar AA, Reşorlu B, Tepeler A, Ziypak T, Söylemez H, Atar M, et al. The forgotten ureteral stent in children: from diagnosis to treatment. J Urol. 2013;189(3):1054-60.

DOI: http://10.1016/j.juro.2012.09.089

12. Saltzman B. Ureteral stents. Indications, variations, and complications. Urol Clin North Am. 1988 Aug; 15(3):481-91.

PMID: 3043868

13. Damiano R, Oliva A, Esposito C, De Sio M, Autorino R, D'Armiento M. Early and late complications of double pigtail ureteral stent. Urol Int. 2002;69(2):136-40.

DOI: https://doi.org/10.1159/000065563

14. Dakkak Y, Janane A, Ould-Ismail T, Ghadouane M, Ameur A, Abbar M. Management of encrusted ureteral stents. African Journal of Urology. 2012;18:131-4.

DOI: http://dx.doi.org/10.1016/j.afju.2012.08.013

15. El-Faqih SR, Shamsuddin AB, Chakrabarti A, Atassi R, Kardar AH, Osman MK. Polyurethane internal ureteral stents in treatment of stone patients: morbidity related to indwelling times. The Journal of urology. 1991 Dec;146(6):1487-91. 
16. Dongol UM, Ghimire R. Multimodal management of forgotten encrusted ureteral stents. Journal of Kathmandu Medical College. 2016;5(4):116-9.

DOI: http://dx.doi.org/10.3126/jkmc.v5i4.18607

17. Abdelaziz AY, Fouda WB, Mosharafa AA, Abelrasoul MA, Fayyad A, Fawzi K. Forgotten ureteral stents: Risk factors, complications and management. African Journal of Urology. 2018;24(1):28-33.

18. El Harrech Y, Abakka N, El Anzaoui J, Ghoundale O, Touiti D. Ureteral stenting after uncomplicated ureteroscopy for distal ureteral stones: a randomized, controlled trial. Minimally Invasive Surgery. 2014;2014(892890).

DOI : https:/www.hindawi.com/journals/mis/2014/892890/

19. Chen Y FJ, Yue Y, Zhao Z, Deng T, Wu W, Zeng G. Externalised ureteral catheter versus double-J stent in tubeless percutaneous nephrolithotomy for upper urinary stones: A Systematic Review and Metaanalysis. J Endourol. 2018(April 26).

DOI: https://10.5114/wiitm.2016.64447 PMID: 28194243 